\title{
European research activities towards a future DEMO gyrotron
}

\author{
J. Jelonnek ${ }^{1,2}$, G. Aiello ${ }^{3}$, S. Alberti ${ }^{4}$, K. Avramidis ${ }^{1}$, A. Bertinetti ${ }^{11}$, A. Bruschi ${ }^{5}$, J. Chelis ${ }^{6}$, T. Franke ${ }^{7,8}$, \\ G. Gantenbein ${ }^{1}$, S. Garavaglia ${ }^{5}$, G. Granucci ${ }^{5}$, G. Grossetti ${ }^{3}$, S. Illy $^{1}$, \\ Z.C. Ioannidis ${ }^{1}$, J. Jin $^{1}$, P. Kalaria ${ }^{1}$, G.P. Latsas ${ }^{6}$, H. Laqua ${ }^{9}$, A. Leggieri ${ }^{10}$, F. Legrand ${ }^{10}$, A. Marek ${ }^{1}$,
}

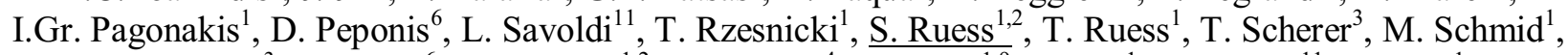

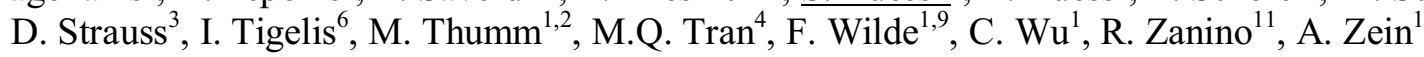

${ }^{1} \mathrm{IHM},{ }^{2} \mathrm{IHE},{ }^{3} \mathrm{IAM}-\mathrm{AWP}$, Karlsruhe Institute of Technology (KIT), D-76131 Karlsruhe, Germany

${ }^{4}$ Swiss Plasma Center (SPC), EPFL, CH-1015 Lausanne, Switzerland

${ }^{5}$ Institute of Plasma Physics "P.Caldirola", National Research Council of Italy, Milan, Italy

${ }^{6}$ NCSRD/National and Kapodistrian University of Athens, Faculty of Physics, Athens, Greece

${ }^{7}$ EUROfusion Consortium, Boltzmannstr. 2, D-85748 Garching, Germany

${ }^{8}$ Max-Planck-Institut für Plasmaphysik, Boltzmannstr. 2, D-85748 Garching, Germany

${ }^{9}$ Max Planck Institut für Plasmaphysik, Teilinstitut Greifswald, Wendelsteinstrasse 1, D-17491 Greifswald, Germany

${ }^{10}$ Thales Electron Devices, 2 rue Marcel Dassault, Vélizy-Villacoublay, F-78141, France

${ }^{11}$ NEMO group, Dipartimento Energia, Politecnico di Torino, 24, c. Duca degli Abruzzi, I-10129 Torino, Italy

Coordinated by the EUROfusion Consortium, several European research institutes are working on fusion technologies towards options for a European DEMOnstration Fusion Power Plant (FPP), as a single step between ITER and a commercial FPP, to deliver net electricity by mid of this century. One of the focus areas is the research on a proper Electron Cyclotron Resonance Heating (ECRH) and Current Drive (ECCD) system for which the fusion gyrotron is one of its major key components [1].

A future FPP will probably require an ECCD operating frequency ranging from $170 \mathrm{GHz}$ up to $240 \mathrm{GHz}$ depending on the DEMO baseline. An RF output power of significantly higher than $1 \mathrm{MW}$ (target: $2 \mathrm{MW}$ ) and a total gyrotron efficiency better than $60 \%$ are required. Multi-purpose operation at multiples of the $\lambda / 2$-resonance frequency of the vacuum window of the gyrotron, hence in leaps of about $30 \mathrm{GHz}$ (e. g. 170 / $204 / 238 \mathrm{GHz}$ ) needs to be considered for plasma start-up, heating and current drive. Optionally for possible steering of the $a b-$ sorption layer the gyrotron shall allow a fast frequency tuning in steps of around $2-3 \mathrm{GHz}$. The R\&D work within the EUROfusion work package "WP HCD EC Gyrotron R\&D and Advanced Developments (AD)" is focusing on all of the named targets.

\section{Verification of the coaxial-cavity technology}

The coaxial-cavity gyrotron is a promising technology for future multi-MW fusion gyrotrons [2]. In [3] a world record RF output power of $2.2 \mathrm{MW}$ at short pulses (ms-range) was demonstrated. Nevertheless, the $2 \mathrm{MW}$ coaxial-cavity technology, already considered for the first installation in ITER earlier [4], is still lacking its proofof-concept regarding long-pulse operation. Major concerns are the proper alignment and thermal loading of the cavity wall and its inner conductor as well as the thermal loading of the collector. Its feasibility shall be finally demonstrated by upgrading the existing KIT $2 \mathrm{MW}$ $170 \mathrm{GHz}$ short-pulse pre-prototype to pulse lengths up to $1 \mathrm{~s}$ [5]. In parallel, work is ongoing in the field of advanced cooling concepts [6, 7]. Additionally, two new coaxial-cavity Magnetron Injection Guns (MIGs) are under manufacturing. The first is employing an advanced emitter technology whose major element is a new non- emissive coating. That will significantly reduce the velocity spread of the electrons at the emitter [8]. Secondly, a newly designed Inverse Magnetron Injection Gun (IMIG) will allow for a significant larger emitter radius and therefore increased output power at operating frequencies significantly above $200 \mathrm{GHz}$ by keeping the same or even smaller size of the bore hole of the gyrotron SC magnet [9].

\section{Studies towards a $240 \mathrm{GHz}$ gyrotron}

A frequency up to $240 \mathrm{GHz}$ was selected for the theoretical research work towards a future FPP, considering the requirements for "multi-purpose" and "fast frequency step-tunable" operation at high-field tokamaks and for a wide range of RF beam steering. The coaxialcavity gyrotron technology, and, as a possible fallback solution, the conventional hollow-cavity gyrotron are under investigation. Both technologies were studied regarding to the maximum achievable output power versus efficiency and stability in operation due to tolerances. A generic design strategy was developed to find the optimum operating mode for the two different cavity topologies [10]. Operating scenarios close to $2 \mathrm{MW}$ for the coaxial-cavity technology and around 1.5 MW for the conventional cavity technology have been found in the theoretical analyses (ref. [11-14]).

\section{Advances in Window Technologies}

Fundamental for frequency-step tunable operation is the utilization of a proper broadband window technology. At KIT the CVD-Diamond Brewster-angle window is the favorite. The successful operation of a step-frequency tunable $1 \mathrm{MW}$ short-pulse (ms) gyrotron with a synthetically manufactured diamond Brewster angle output window was demonstrated earlier [15]. Nevertheless, for future DEMO gyrotrons the development of diamond discs of larger size for higher power capabilities, advanced cooling and brazing technologies are mandatorily required and pushed forward at KIT $[16,17]$.

\section{Towards a total efficiency of higher than $60 \%$}

A DEMO gyrotron will require a total efficiency of above $60 \%$ to minimize the electrical power requirements, and, ultimately the recirculating power in the bal- 
ance of plant. Considering an interaction efficiency of typical $35 \%$ between the electron beam and the microwave, a large fraction of the input energy remains in the spent electron beam. Up to $60 \%$ of the spent beam energy might be recovered by a single-stage depressed collector (SDC) which will lead to an overall gyrotron efficiency of about $50 \%$ in theoretically best case. An overall efficiency of higher than $60 \%$ requires the use of advanced multi-stage depressed collectors (MDCs). Two concepts are under investigation [18] at KIT. In the first one, the gyrotron magnetic field is unwound utilizing wellcontrolled non-adiabatic transitions, whereas in the second one, the electrons are sorted by an $\mathrm{E} \times \mathrm{B}$ drift [19-22].

\section{Intelligent control systems for gyrotrons}

In future, intelligent control systems shall allow higher output powers of gyrotrons by reducing the necessary safety margins, hence allowing for an operation close to the stability limits [23]. Firstly applied to the W7-X gyrotrons, a new control concept is under investigation. The idea is to detect the stray radiation caused by the excitation of parasitic modes at the stability limits and to use that as an indicator for the operational stability of the W7-X gyrotrons. A feedback control system is under development.

\section{Advanced gyrotron tests environments}

In 2015, the final design review and procurement of the High-Voltage Power Supply (HV PS) for the new KIT FULGOR gyrotron teststand started [24]. FULGOR will allow $\mathrm{CW}$ operation of gyrotrons with a required input power of 5 ( $2^{\text {nd }}$ phase: 10) MW DC. A $10.5 \mathrm{~T} \mathrm{SC}$ magnet will allow the operation of gyrotrons up to $240 \mathrm{GHz}$. That includes the upgrade of all the measurements systems, which have been developed for verification of the frequency spectrum, calorimetry and quasioptical transmission $[25,26]$.

\section{Acknowledgement}

This work was carried out within the framework of the EUROfusion Consortium and have received funding from the Euratom research and training programme 2014-2018 under grant agreement No. 633053. The views and opinions expressed herein do not necessarily reflect those of the European Commission.

\section{References}

1. G. Granucci et al. The EC-system of EU DEMO: concepts for a reactor heating system // SMP, 2017, NhizhnyNovgorod.

2. B. Piosczyk et al. $165-\mathrm{GHz}$ coaxial cavity gyrotron // IEEE Trans. Plasma Science, 2004, Vol. 32, No. 3, 413-417.

3. T. Rzesnicki, et al. 2.2-MW Record Power of the 170-GHz European Pre-Prototype Coaxial-Cavity Gyrotron for ITER // IEEE Trans. Plasma Science, 2010, Vol. 38, No. 6, pp. 1141-1149.

4. J.-P. Hogge et al The European $2 \mathrm{MW}, 170 \mathrm{GHz}$ coaxial cavity gyrotron for ITER // Joint 32nd IRMMW/15th Int. Conf. on THz Electronics, 2007, Cardiff.

5. S. Ruess et al. Design and Manufacturing Process for the KIT 2-MW 170-GHz Coaxial-Cavity Longer-Pulse Gyrotron // SMP, 2017, Nhizhny-Novgorod.
6. Bertinetti et al. Multi-physics analysis of a $1 \mathrm{MW}$ gyrotron cavity cooled by mini-channels // presented at SOFT, 2016, P1.029, accepted for publication in Fus. Eng. Des., 2017.

7. K. Avramidis et al. Numerical studies on the influence of cavity thermal expansion on the performance of a high-power gyrotron // IVEC, London, 2017.

8. I. Pagonakis et al. Influence of emitter ring manufacturing tolerances on electron beam quality of high power gyrotrons, // Physics of Plasmas, 2016, 23, 083103, doi: 10.1063/1.4959113.

9. S. Ruess et al. An Inverse Magnetron Injection Gun for the KIT 2-MW Coaxial-Cavity Gyrotron // IEEE Trans. Electron Devices, 2016, Vol. 63, pp. 2104-2109.

10. J. Franck, et.al. A generic mode selection strategy for high-order mode gyrotrons operating at multiple frequencies // Nucl. Fusion, 2014, Vol. 55, No. 1.

11. J. Franck, et. al. Direct voltage depression calculation of arbitrary electron beams in misaligned coaxial gyrotron cavities // IEEE Trans. on Electron Devices, 2016, Vol. 63, No. 9.

12. J. Franck, et.al. Insert misalignment in coaxial gyrotrons: physical effects and numerical treatment // 5th ITG Int. Vacuum Electronics Workshop, 8 - 9, Sept. 2016, Bad Honnef, Germany.

13. P. Kalaria, et. al. RF behavior and launcher design for a fast frequency step-tunable $236 \mathrm{GHz}$ gyrotron for DEMO // Journal of RF-Engineering and Telecommunications - Frequenz, 2016, Vol. 71, No. 3-4, pp. 161-171.

14. P. Kalaria, et. al. Systematic cavity design approach for a multi-frequency gyrotron for DEMO and study of its RF behavior // Physics of Plasmas, 2016, Vol. 23, No. 9.

15. G. Gantenbein et al. First operation of a step-frequency tunable 1-MW gyrotron with a diamond Brewster angle output window // IEEE Trans. Electron Devices, 2014, Vol.61, No. 6, pp.1806-1811.

16. G. Aiello et al. CVD diamond Brewster window: feasibility study by FEM analysis // Proc. Workshop on Electron Cyclotron Emission and Electron Cyclotron Resonance Heating, Deurne, 2012.

17. G. Aiello et al. Cooling concepts for the CVD diamond Brewster-angle window // IRMMW-THz 2017.

18. C. Wu et al. Comparison between Controlled NonAdiabatic and ExB Concepts // SMP, 2017, Nhizhny-Novgorod.

19. I. Pagonakis et al. A New concept for the collection of an electron beam configured by an externally applied axial magnetic field // IEEE Trans. Plasma Sci., 2008, vol. 36, no. 2, pp. 469-480.

20. I.Gr. Pagonakis et al. Multistage depressed collector conceptual design for thin magnetically confined electron beams // Physics of Plasmas, 2016, 23, 043114.

21. C. Wu et al. Conceptual designs of $\mathrm{E} \times \mathrm{B}$ multistage depressed collectors for gyrotrons // Physics of Plasmas, 2017, Vol. 24, No. 4, 043102.

22. C. Wu et al. Novel multistage depressed collector for high power fusion gyrotrons based on an E $\times$ B Drift concept // IVEC, 2017, London.

23. F. Wilde et al. Measurements of satellite modes in 140 $\mathrm{GHz}$ Wendelstein 7-X gyrotrons: an approach to an electronic stability control // IVEC, 2017, London.

24. M. Schmid et al. The $10 \mathrm{MW}$ EPSM modulator and other key components for the KIT gyrotron test facility FULGOR // Fus. Eng. Des., 2017, https://doi.org/10.1016/ j.fusengdes.2017.02.035.

25. M. Losert et al. RF Beam Measurements of QuasiOptical Mode Converters in the mW Range // IEEE Trans. Plasma Sci., 2013, Vol. 41, No. 3, pp. 628-632.

26. A. Schlaich et al. Time-dependent spectrum analysis of high power gyrotrons // KIT Sci. Publishing, 2015, IHM. 